\title{
Erster Meilenstein für die neue Landesbibliothek
}

Der 18. November 2015 war für die Landesbibliothek ein besonderer Tag. An diesem sonnigen Herbsttag - vielleicht ein gutes Omen - erfolgte mit der Grundsteinlegung der symbolisch wichtige "Startschuss" für die eigentlichen Arbeiten an dem neuen Erweiterungsbau.

Über 120 Gäste fanden sich im Beisein von Finanzund Wirtschaftsminister Nils Schmid, Staatssekretär Jürgen Walter (Ministerium für Wissenschaft, Forschung und Kunst) und dem Stuttgarter Baubürgermeister Peter Pätzold auf der Baustelle ein, um in feierlichem Rahmen den Grundstein für den Erweiterungsbau zu legen.

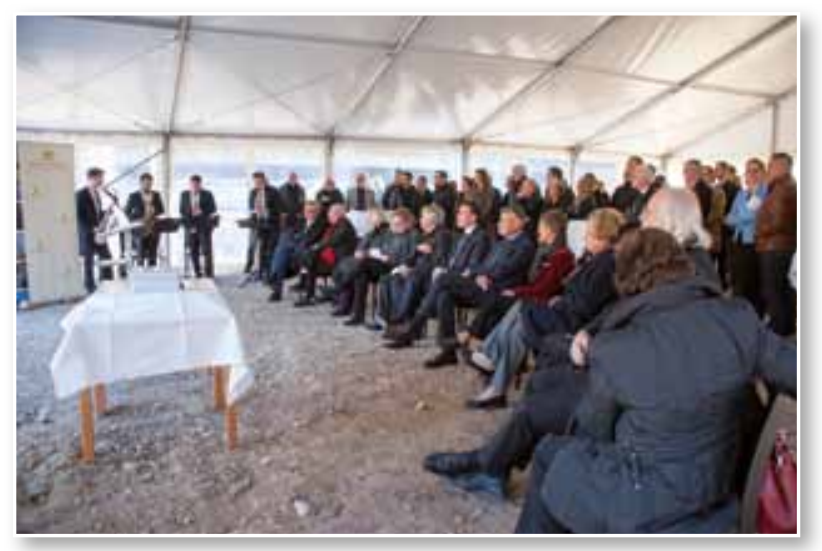

Abb. 1: Feierliche Versammlung zur Grundsteinlegung im Zelt

Das eigens dafür errichtete Zelt in der Baugrube erreichte man über eine Metallleiterkonstruktion. Für die musikalische Umrahmung sorgte das Stutt-

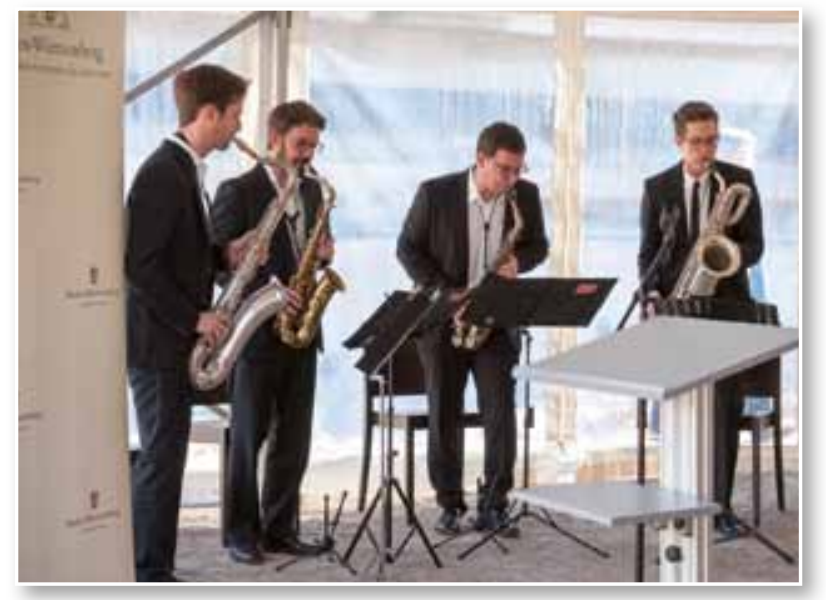

Abb. 2: Saxophon-Quartett SüdSax garter Saxophon-Quartett SüdSax, das gemeinsam an der Musikhochschule studiert hat. Es begleitete die Veranstaltung mit einer stimmungsvollen Mischung aus Jazz und Unterhaltungsmusik.

Die Begrüßung erfolgte durch die Bauherrin Annette Ipach-Öhmann, Leiterin des Landesbetriebs Vermögen und Bau Baden-Württemberg.

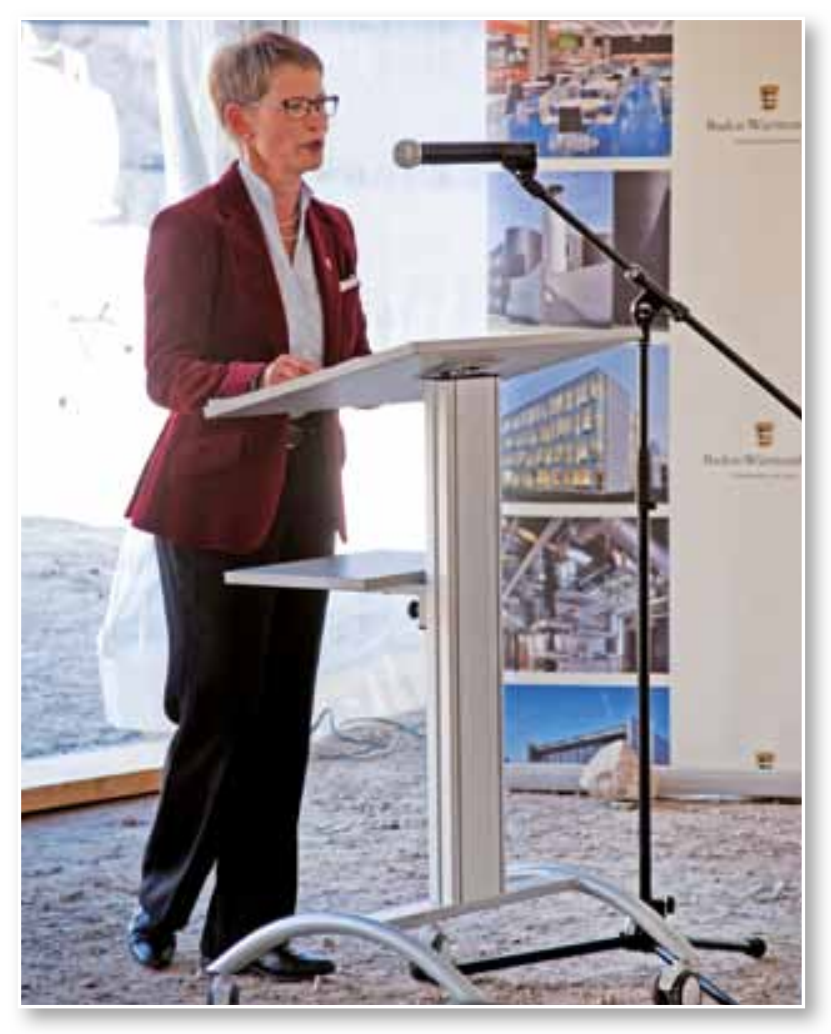

Abb. 3: Bauherrin Annette Ipach-Öhmann

Für Finanz- und Wirtschaftsminister Nils Schmid war es ein wichtiger Anlass, um noch einmal die Bedeutung der neuen Landesbibliothek als "Gedächtnis des Landes" zum Ausdruck zu bringen. Er hob die "mutige Architektur" hervor und betonte, dass die Investition von 48 Millionen Euro bei diesem "lebendigen Lernort" gut angelegt und auch erforderlich sei. Er versprach auch weiterhin seine Unterstützung bei der Fortführung des Bauprojekts, das er als eine "wichtige und richtige Anlage in die Zukunft unserer Gesellschaft" herausstellte. 


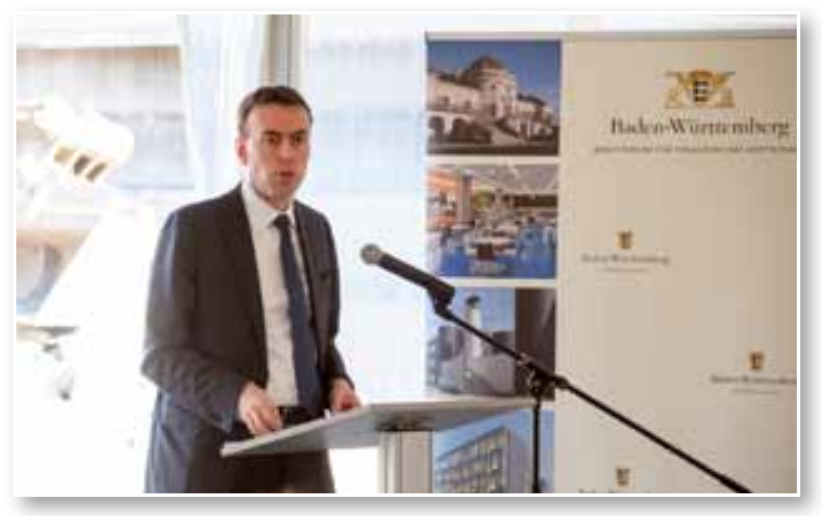

Abb. 4: Finanz- und Wirtschaftsminister Nils Schmid

Staatssekretär Jürgen Walter erinnerte, dass die Landesbibliothek inzwischen an ihre absoluten Kapazitätsgrenzen angekommen sei: „Die größte wissenschaftliche Universalbibliothek braucht dringend mehr Magazinfläche, mehr Arbeitsplätze und den so lange ersehnten Freihandbereich. Die Erweiterung der Württembergischen Landesbibliothek trägt diesem Erfordernis Rechnung. Ich bin froh, dass wir mit der heutigen Grundsteinlegung diesem Ziel einen weiteren wichtigen Schritt näher gekommen sind."

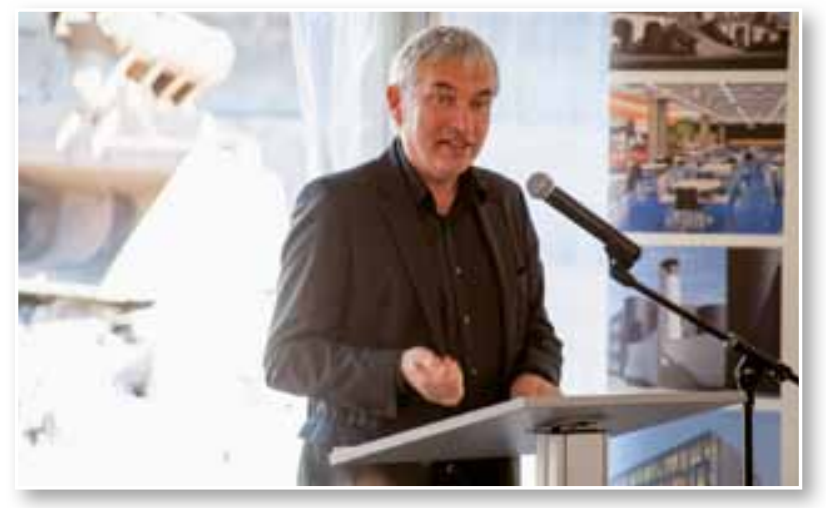

Abb. 5: Staatssekretär Jürgen Walter

\section{Baubürgermeister Peter Pätzold unterstrich die}

Bedeutung der erweiterten Landesbibliothek für die Stuttgarter Kulturmeile, die durch das neue Ensemble deutlich an Attraktivität gewönne.

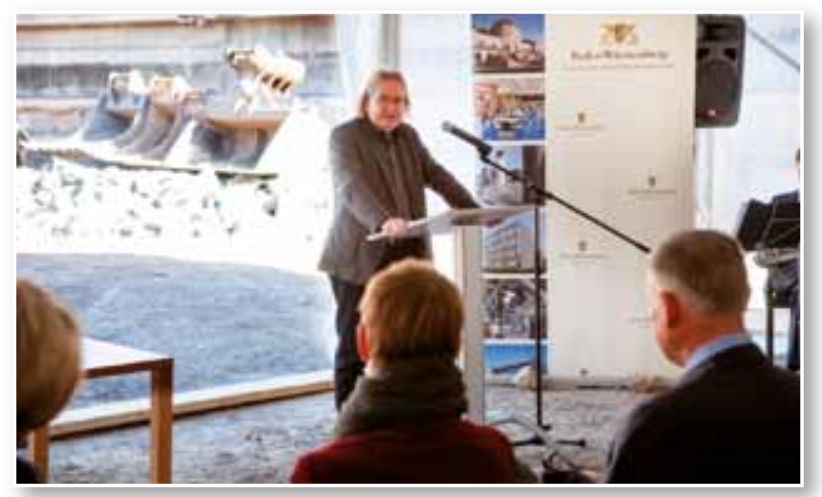

Der Direktor der Württembergischen Landesbibliothek, Dr. Hannsjörg Kowark, wies darauf hin, dass nur nach der erfolgreichen Sanierung und Umnutzung des Bestandsgebäudes sich wirklich alle ersehnten Ziele gänzlich erfüllten, da Alt- und Neubau als eine Einheit betrachtet werden müssen. Die unumgängliche Asbestsanierung erfordere leider die zeitweise Auslagerung der Magazinbestände. Als einzige sinnvolle ökonomische Möglichkeit nannte er die Tiefgarage, die vorübergehend die 3,5 Millionen Bücher aufnehmen könnte. Diese Möglichkeit dürfe nicht verpasst werden, so dass schon früh die Weichen in diese Richtung gestellt werden müssten.

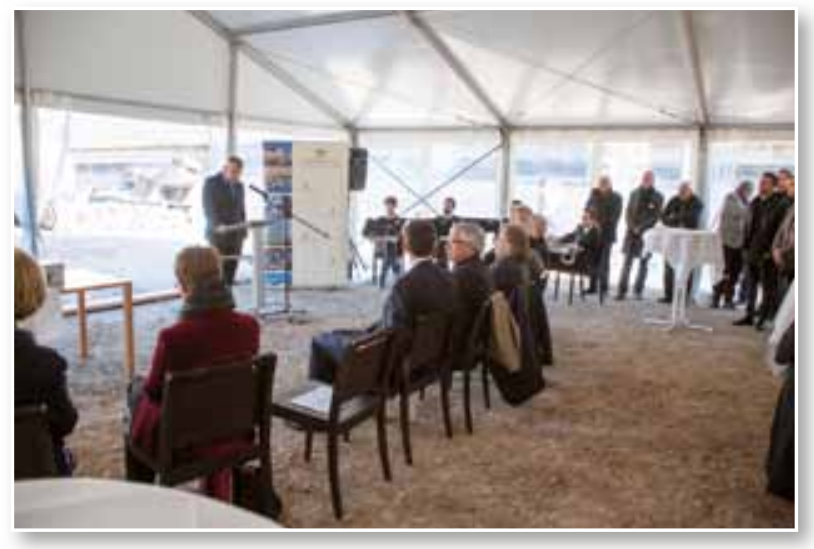

Abb. 7: Hannsjörg Kowark, Ltd. Direktor der WLB

Für Finanzminister Nils Schmid war diese Option gleichfalls die vernünftigste Lösung.

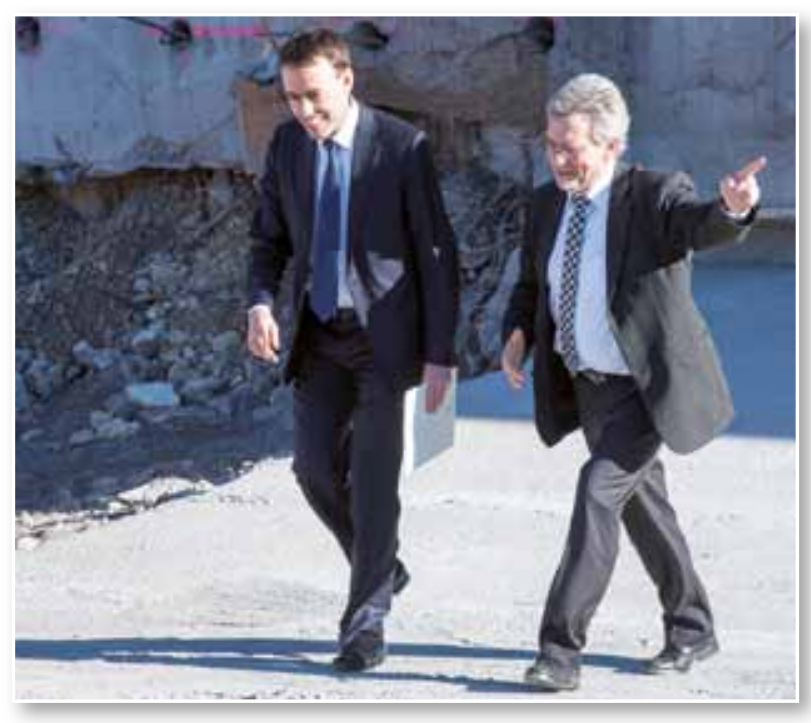

Abb. 8: Nils Schmid mit Roland Wenk (Amtsleiter Vermögen und Bau BW)

Nach den Reden wurde anstelle der typischen Zeitkapsel ein Metallkoffer gefüllt: 


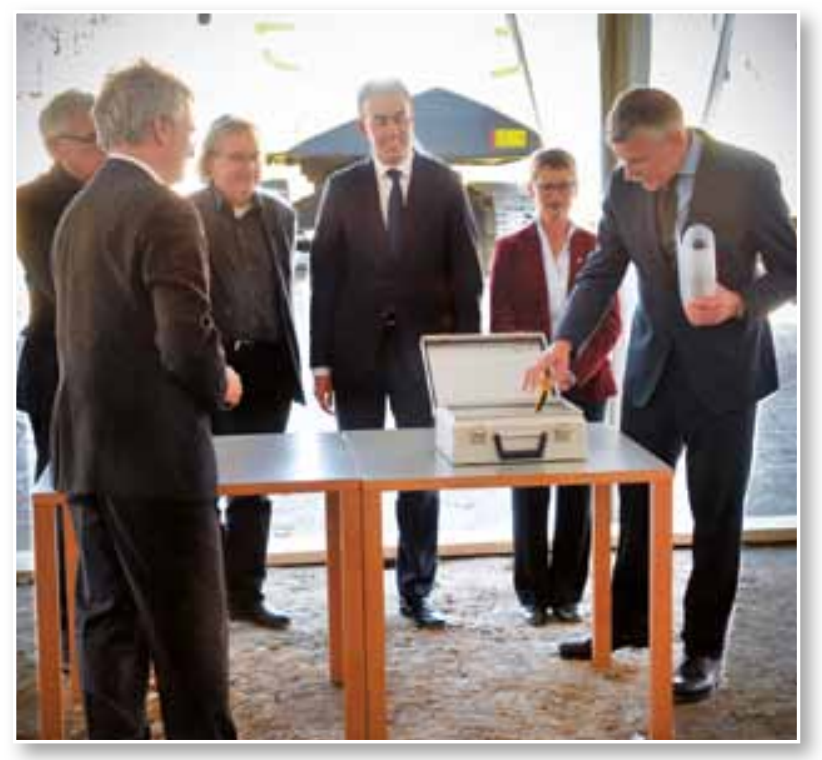

Neben dem Jubiläumsband zur Ausstellung „Carl Eugens Erbe“ sollten der Bauantrag, die Baugenehmigung, eine Tageszeitung vom 18. November, eine Millenium-Disk mit dem Katalog der WLB sowie eine Flasche Lauffener Hölderlinwein (Jahrgang 2008) an diesen Tag erinnern. Der Koffer wurde anschließend in einer feierlichen Zeremonie versenkt. Ein Stehempfang rundete die gelungene Veranstaltung ab.

Jörg Ennen

Abb. 9: Das feierliche Befüllen des Metallkoffers

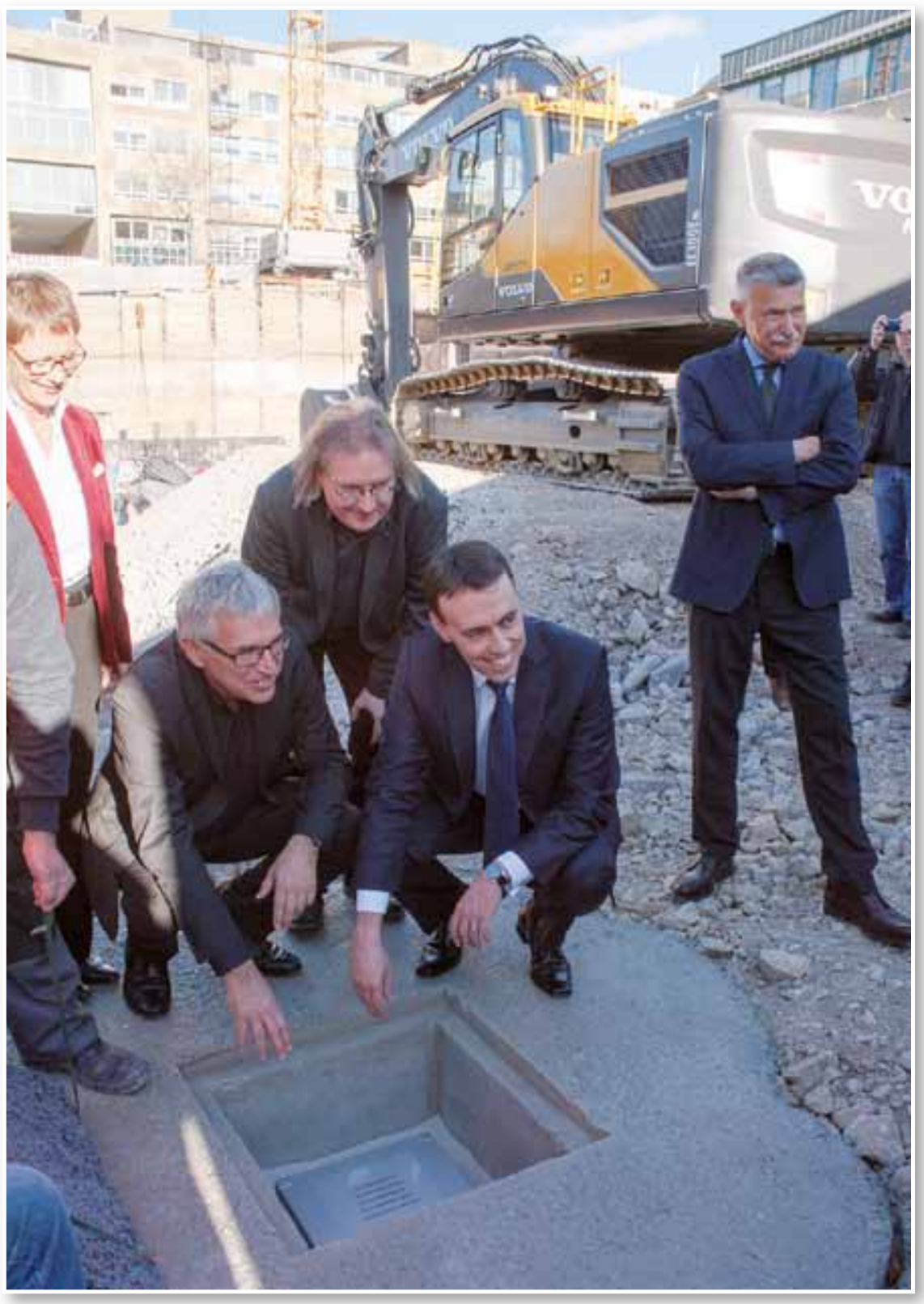

